Aus der Universitäts-Frauenklinik in Kiel.

(Direktor: Geh. Med.-Rat Prof. Dr. Pfannenstielt.)

Ueber Chlornatriumentziehung bei Erkrankungen der Harnwege während der Schwangerschaft.

Von Dr. Oskar Jaeger, Assistent der Klinik.

Vor einiger Zeit hat Cramer die Einschränkung der Kochsalzzufuhr zur Behandlung des Hydrops graviditatis warm empfohlen, da es ihm gelungen war, durch eine entsprechende Diät einfache Schwangerschaftsödem $\theta$ in kurzer Zeit zum Schwinden zu bringen. Seinen Ausführungen trat Birnbaum entgegen, der experimentell feststellte, daß eine Chlornatriumretention im Körper bei einfachem Schwangerschaftshydrops ohne Eiweißausscheidung de facto nicht nachzuweisen ist. Nur bei gleichzeitig bestehender Schwangerschaftsalbuminurie konnte Birnbaum eine erheblichere Verminderung der Koch-

1) Uhle $\mathrm{nhuth}$ und Mate ufe 1, Ueber die Wirkung von atoxylsaurem Quecksilber bei Spirochätenkrankheiten, insbesundere bei der experimentellen Syphilis. Medizinische Klinik 1908, No. 43. 
salzausscheidung durch die Nieren gegenüber der Norm konstatieren.

Der Frage des Kochsalzstoffwechsels bei Nierenkranken überhaupt haben sich in den letzten Jahren besonders die Internisten mit erhöhtem Interesse zugewandt, aber trotz zahlreicher Stoffwechselversuche und trotz eingehender klinischer Beobachtungen sind die Ansichten der Autoren über die Beziehungen zwischen Nephritis und Kochsalzausscheidung durchaus nicht übereinstimmend. Während auf der einen Seite eine verminderte Ausscheidung der Chloride durch die erkrankten Nieren und eine Anhäufung derselben in den Körperorganen mehrfach festgestellt wurde (Achard, Widal, Strauss, Bohne u. a.), konnten diese Beobachtungen von anderen Untersuchern nicht bestätigt werden (Bittorf und Jochmann, Bittorf). Dementsprechend mußte auch das Abhängigkeitsverhältnis der nephritischen Oedeme von der Chlornatriumretention eine verschiedenartige Bewertung finden. Bittorf lehnte es auf Grund seiner Experimente überhaupt ab, die primäre Kochsalzretention als eine wesentliche Ursache für die Oedembildung Nierenkranker anzuerkennen; ihm schienen vielmehr GefäBschädigungen und primäre Wasserrentention die Hauptrolle dabei zu spielen. Einen ähnlichen Standpunkt für die Nephritis gravidarum vertrat $Z$ angemeister. Im Gegensatz hierzu war es in Deutschland besonders Strauss, der gerade der NaCl-Retention für die Entstehung nephritischer Oedeme eine hohe Bedeutung zuschrieb und therapeutisch für diejenigen Fälle von Nierenentzündung, die eine Neigung zu Hydropsien erkennen lassen, die, wie or sich ausdrückt, eine „Hydropsietendenz" zeigen, Chlorentziehungskuren empfahl. Die Verhandlungen auf dem letzten Kongreß für innere Medizin haben gezeigt, dab die Internisten wohl in der Hauptsache auf dem eben skizziorten Standpunkt von Strauss stehen. Einen Bewois für die Richtigkeit der Straussschen Lehre, wenigstens für gewisse Fälle, liefert, eine Beobachtung von Widal, dem es gelang, die Oedeme eines Nephritikers beliebig oft zum Schwinden zu bringen, sobald er ihm eine kochsalzarme Kost gab, und bei dem sich sofort die Oedeme wieder einstellten, wenn zu dieser Diät $10-12 \mathrm{~g} \mathrm{NaCl}$ pro die zugesetzt wurden.

In der Nephritis gravidarum haben wir nun eine Nierenschädigung vor uns, die im allgemeinen mit recht erheblichen Oedemen einherzugehen pflegt und bei der schon aus diesem Grunde die therapeutischeVerwendung einer kochsalzarmen Diät gerechtfertigt erscheint. Es ist dies um so mehr der Fall, als ja tatsächlich bei der Schwangerschaftsalbuminurie die NaClAusscheidung durch die Nieren herabgesetzt ist. Allerdings sind auch die Ansichten über die Bedeutung und das Wesen der bestehenden Kochsalzretention noch geteilt (Birnbaum, Zangemeister).

Was zunächst die praktische Durchführung einer kochsalzarmen Diät betrifft, so haben wir uns dabei von folgenden Gesichtspunkten leiten lassen: $\mathrm{Zu}$ vermeiden sind alle Speisen, die bereits im rohen Zustande einen größeren Salzgehalt aufweisen, oder deren schmackhafte Zubereitung mit einem Zusatz von größeren Kochsalzmengen notwendig verbunden ist. Aus diesem Grunde haben wir z. B. auf die Darreichung von Fleisch und Fleischbrühe überhaupt ganz verzichtet. Die einfachste Form der salzarmen Ernährung stellt die reine Milchdiät dar, denn die Kuhmilch enthält nur etwa $0,15-0,18 \%$ Kochsalz. Neben manchem Vorzug, den die absolute Milchdiät bietet, haftet ihr aber der Nachteil an, dab sie mit der Darreichung gröberer Flïssigkeitsquantitäten verbunden ist, was für die in ihrer Funktion schwer geschädigten Nieren nicht gleichgültig sein kann. Außerdem gibt es eine große Anzahl Menschen, bei denen eine konsequent durchgeführte Milchdiät sehr. bald so heftigen Widerwillen hervorruft, dab sie lieber auf jede Nahrungsaufnahme verzichten, als sich länger den bestehenden Diätvorschriften unterwerfen. Wir sind infolgedessen gezwungen, uns nach anderen kochsalzarmen Nahrungsmitteln umzusehen, um mehr Abwechslung in die Kostordnung $\mathrm{zu}$ bringen. Die für den Körper notwendigen Eiweißmengen führen wir in der Form des Eiereiweißes ein; es lassen sich die verschiedensten Eierspeisen (Setzei, Rührei, Omelette) ohne jeden Salzzusatz herstellen und durch Hinzufügen von entsprechenden Mengen Zuckers recht schmackhaft gestalten.
Butter geben wir reichlich, aber nur in ungesalzenem Zustand. Kohlehydrate werden in folgenden Formen gereicht: Als Mehlspeisen, Reis (Milchreis), Gries, Sago, Kartoffeln (besonders als Bratkartoffeln), Puddings, Schleimsuppen mit reichlichem Zusatz von Butter und Zucker; dazu etwas Weißbrot. Von Gemüsen bevorzugen wir wegen ihres geringen Salzgehaltes besonders Blumenkohl, grüne Erbsen, Pilze, Karotten. Der Nährwert der Gemüse läßt sich ganz erheblich durch Zugabe von Mehl und vor allem von größeren Mengen Butter noch erhöhen. Obst in frischem und gekochtem Zustand vervollständigt den Küchenzettel. Von Getränken kommen in Betracht: Wasser, Limonaden, Malzkaffee, leichter Tee, Milch.

An der Hand derartiger Diätvorschriften läßt sich mühelos eine kochsalzarme Kost herstellen, die abwechselungsreich genug ist, um wochenlang ohne größere Schwierigkeiten von seiten der Patienten durchgeführt werden $\mathrm{zu}$ können. Durch regelmäbige Wägungen überzeugen wir uns, daß unsere Kranken nicht unterernährt werden.

Nach diesem Regime haben wir seit etwa Jahresfrist alle Patientinnen mit Nephritis gravidarum und Eklampsie ernährt. Aber auch bei Frauen, die an Cystitis oder Pyelitis litten, hatten wir öfter Gelegenheit, die kochsalzarme Diät anzuwenden. In diesen letzten Fällen waren die leitenden Gesichtspunkte dabei allerdings andere als bei der Nephritis gravidarum und Eklampsie. Ich werde noch darauf zurückkommen.

\section{Nephritis gravidarum-Eklampsie.}

Die Schwere der einzelnen Krankheitsfälle, bei denen die kochsalzarme Kost therapeutisch angewendet wurde, war sehr verschieden. Meist handelte es sich um weit vorgeschrittene Formen der Nephritis gravidarum, mit hochgradigen Oedemen, starker Albuminurie und Zylindrurie, hohem Blutdruck und schlechtem Allgemeinbefinden, oder um bereits ausgebrochene Eklampsien. Daneben fanden sich aber auch leichtere Krankheitsbilder mit nur geringer Eiweißausscheidung, geringen Oedemen und fast ungestörtem Wohlbefinden. In sämtlichen Fällen wurde die kochsalzarme Diät mit gleicher Schärfe durchgeführt.

Bei den klinisch-leichteren Schwangerschaftsnephritiden ist es unser Bestreben, den Krankheitsprozeb womöglich ganz zu beseitigen oder wenigstens eine Verschlimmerung des Leidens, wie sie gegen Ende der Gravidität ganz regelmäßig einzutreten pflegt, nach Möglichkeit zu verhüten. In allen diesen Fällen hat uns unsere Therapie niemals im Stich gelassen, sodab wir das normale Endo der Schwangerschaft abwarten konnten und bei der Geburt ein ausgetragenes, lebenskräftiges Kind erzielten. Nur ausnahmsweise kam es zu einer vorzeitigen Spontangeburt. Für das Gesagte diene folgender Fall als Paradigma:

Frau J., V-para, kommt wegen zunehmender Schwellung beider Beine in die Klinik. Es besteht eine Schwangerschaft, die etwa dem achten Schwangerschaftsmonat entspricht. Mäßige Oedeme der unteren Extremitäten, geringes Bauchdeckenödem. Der Urin enthält bei der Aufnahme $5 \%$ Albumen und zahlreiche hyaline und granulierte Zylinder. Blutdruck 145 (gemessen mit dem Sphygmomanometer von Riva Rocci-Recklinghaugen), ist also zweifellos erhöht. Allgemeinbefinden gut. Therapie: Bettruhe, kochsalzarme Diät, feuchte Einpackungen, keine Diuretica.

Nach etwa zwei Wochen sind die Oedeme völlig geschwunden, der Eiweißgehalt des Urins sinkt allmählich und schwankt bis zur Entbindung zwischen 2 und $3 \%$. Die Zylindrurie hört völlig auf. Der Blutdruck zeigt normale Werte $(110-120 \mathrm{~mm} \mathrm{Hg})$.

24 Tage nach der Aufnahme in die Klinik setzen Wehen ein, und es erfolgt eine normale Entbindung. Unter dem Einfluß des Geburtstraumas steigt der Eiweibgehalt deg Urins auf $6 \%$. Im Sediment finden sich wieder sehr zahlreiche Zylinder. Neun Tage post partum ist die Eiweißausscheidung auf $1 \%$ gesunken. Zylinder sind nicht mehr nachzuweisen. Das neugeborene Kind wog $2180 \mathrm{~g}$ und war $46 \mathrm{~cm}$ lang.

In diesem speziellen Falle ist es uns also gelungen, die bestehende Nephritis gravidarum lediglich durch kochsalzarme Diät und durch Bettruhe ganz erheblich zu bessern. Die Gravidität dauerte von dem Beginn der Behandlung ab noch $31 / 2$. Wochen; die Geburt trat aber doch noch einen Monat $z u$ früh ein. Für das Leben des Kindes waren die gewonnenen $3 \frac{1}{2}$ Wochen von der allergrößten Bedeutung, denn es wurde 
durch diese Verlängerung der Schwangerschaft möglich, ein zwar kleines und nicht völlig ausgetragenes, aber doch lebensfähiges Kind mit guten Lebenschancen zu erzielen.

Gerade in solchen Fällen, in denen unter Umständen der Gewinn einer Woche die Lebensfrage des Kindes entscheidet, verdient aber jede therapeutische Maßnahme ernste Beachtung, die uns eine Heilung oder Besserung der Schwangerschaftsnephritis in Aussicht stellt.

In den schweren und schwersten Fällen der Nephritis gravidarum werden wir eine Restitutio ad integrum vor der Entbindung wohl mit keiner der üblichen konservativen Behandlungsmethoden erreichen. Wir müssen uns vielmehr damit begnügen, eine Besserung des gesamten Krankheitsbildes zu erstreben, und können erst nach der Geburt eine völlige Ausheilung der geschädigten Nieren erwarten. Bisweilen werden wir nicht einmal dieses Ziel erreichen; der klinische Verlauf der Krankheit läBt vielmehr eine weitere Verschlechterung erkennen, und es bildet sich in kurzer Zeit ein Zustand heraus, der den Ausbruch des ersten eklamptischen Anfalls jeden Augenblick befürchten läßt. Diese schweren Fälle noch weiterhin konservativ behandeln zu wollen, würde eine ernste Gefährdung des Lebens der betreffenden Kranken bedeuten. Die sofortige Einleitung der künstlichen Frühgeburt ist hier unbedingt geboten.

Wir haben es uns daher bei der Behandlung jeder schweren Nephritis gravidarum zur Regel gemacht, zunächst einen Versuch mit der konservativen Therapie zu machen, indem wir Bettruhe, salzfreie Diät, feuchte Einpackungen, Diuretica etc. verordnen. Verschlechtert sich aber der allgemeine Zustand, steigt der Blutdruck, nehmen die Oedeme, die Albuminurie, die Zylindrurie $\mathrm{zu}$, tritt Blut im Urin auf, stellen sich schwere Veränderungen am Augenhintergrund ein, dann zögern wir nicht länger und leiten unverzüglich künstlich die Geburt ein.

Fragen wir uns nun nach der Leistungsfähigkeit unserer konservativen Therapie, so können wir feststellen, dafl der Erfolg auch in diesen klinisch schweren Fällen von Nephritis gravidarum recht häufig durchaus befriedigend war. Garnicht selten verschwanden selbst hochgradige 0edeme in wenigen Tagen völlig, die Albuminurie und Zylindrurie besserten sich erheblich, der Blutdruck sank, und das schlechte Allgemeinbefinden der Kranken hob sich in kurzer Zeit.

Die kurze Wiedergabe einer derartigen Krankenbeobachtong erhärte das eben Gesagte: Fr. Tr., 29 Jahre, I. Gravidität, suchte die Klinik auf, weil bei ihr innerhalb der letzten 14 Tage sehr starke Schwellungen am ganzen Körper aufgetreten waren. Es bestand eine Gravidität im zehnten Monat. Die Oedeme waren besonders stark an den unteren Extremitäten und im Gesicht. Eiweißgehalt des Urins etwa $20 \%$, im Sediment hyaline und granu. lierte Zylinder, Blutdruck 175. Heftige Schmerzen im Verlauf der großen Nervenstämme des linken Beines. Therapie: Bettruhe, salzfreie Diăt, feuchte Packung.

Bereits nach fünf Tagen waren die Oedeme spurlos verschwunden. Die Urinmengen waren dabei nicht vermehrt $(800-1000 \mathrm{ccm}$ pro die), wohl aber schwitzte die Patientin ganz ungewöhnlich stark, nicht nur während der Packungen, sondern auch spontan; der Eiweißgehalt des Urins war bereits nach zwei Tagen auf $5 \%$ gesunken und erreichte nie wieder die ursprüngliche Höhe. Die Zylindrurie nahm gleichfalls ab; der Blutdruck sank allmählich und betrug am neunten Tage nach der Aufnahme noch $158 \mathrm{~mm} \mathrm{Hg}$.

Am zehnten Tage erfolgte die spontane Geburt eines leicht mazerierten, sicher luetischen Kindes (Spirochätenbefund positiv). Bei der Mutter fehlte jedes Symptom einer bestehenden Lues. Wassermannsche Reaktion negativ.

Intra partum Albumengehalt des Urins $9 \%$. Reichliche $\mathrm{Zy}_{-}$ linder, Blutdruck 185 . Fortsetzung der salzfreien Diät im Wochenbett.

Befund zwölf Tage post partum: allgemeines Befinden ausgezeichnet, keine Oedeme. Geringe Albuminurie $(1 \%)$, einzelne hyaline Zylinder im Harnsediment. Blutdruck 135. Wochenbett ohne Besonderheiten.

Derartig eklatante Erfolge der NaCl-Entziehung bei der Behandlung schwerer und schwerster Schwangerschaftsnephritiden haben wir wiederholt gesehen, womit der praktische Wert der Methode auch für diese vorgeschrittenen Fälle erwiesen sein dürfte. Besonders bemerkenswert ist es, daf die rasche Beseitigung der Oedeme ohne jede Darreichung eines diuretisch wirkenden Medikamentes erreicht werden konnte. Aber auch alle andern Krankheitssymptome gingen erheblich zurück, sodab die von verschiedenen Seiten geäuferten Befürchtungen, es könnten die Nieren durch die Einschränkung der NaClZufuhr direkt geschädigt werden, de facto nicht zuzutreffen scheinen.

Als ein in geeigneten Fällen sehr prompt wirkendes Mittel zur raschen Entwässerung des Körpers haben wir die mit der kochsalzarmen Diät kombinierte stärkere Einschränkung der Wasserzufuhr kennen gelernt. Es ist ja selbstverständlich, daB es bei jeder Nephritis gravidarum vermieden werden mub, größere Flüssigkeitsmengen zu verabreichen, um die erkrankten Nieren nach Möglichkeit zu entlasten. Aus diesem Grunde konzedieren wir unseren Patientinnen durchschnittlich nicht mehr als etwa $1200-1500 \mathrm{ccm}$ Flüssigkeit pro die. Ich habe auch bereits darauf hingewiesen, dâ die an sich kochsalzarme Milchdiät gerade wegen der mit ihr verbundenen gesteigerten Wasserzufuhr in letzter Zeit therapeutisch von uns nicht mehr verwendet wurde; wohl aber haben wir von der sogenannten Karell-Kur bisweilen Gebrauch gemacht. Diese Kur stellt bekanntlich eine reine Milchdiät mit gleichzeitiger Einschränkung der Flüssigkeitszufuhr dar (viermal täglich je $200 \mathrm{~g}$ Milch), die sich bisweilen auch dann noch als recht brauchbar erwiesen hat, wenn alle andern Behandlungsmethoden versagt hatten.

Ein in dieser Beziehung charakteristisches Beispiel betraf eine 37jährige III-para, die an schwerer Nephritis gravidarum mit sehr ausgedehnten Oedemen litt. Post partum sank zwar der Eiweißgehalt des Urins von $12 \%$ auf $4 \%$, aber die Entwässerung des Körpers wollte nicht recht in Gang kommen. Vom dritten Wochenbettstage ab Karell-Kur. Daraufhin sehr starke Diurese, soda $B$ das tägliche Urinquantum in der folgenden Zeit die eingeführte Flüssigkeitsmenge durchschnittlich um einen Liter übertraf. Die Oedeme waren nach einer Woche vőllig verschwunden.

In der Praxis läßt sich jedoch die Karell-Kur nur in Ausnahmefällen durchführen, da sie von den Kranken so grobe Entbehrungen fordert, daf deren Ausdauer bald zu erlahmen beginnt. Auferdem ist sie mit einer derartigen Unterernährung verbunden, då ihre Anwendung für längere Zeit nicht geraten erscheint.

Wie bereits erwähnt, haben wir nun versucht, unsere kochsalzarme Kost gleichfalls mit einer stärkeren Einschränkung der Flüssigkeitszufuhr zur Beseitigung hartnäckiger Oedeme zu kombinieren. Die erzielten Resultate waren recht bemerkenswert, wie unter anderen folgender Fall beweisen mag.

Bei einer III-para mußte die Schwangerschaft wegen schwerster, rasch fortschreitender Nephritis gravidarum künstlich unterbrochen werden. Der Urin enthielt durchschnittlich $10 \%$ Eiweiß. Im Sediment fanden sich enorme Mengen hyaliner und granulierter Zylinder. Die tägliche Urinmenge betrug ante partum etwa 200 bis $300 \mathrm{ccm}$. Dabei bestanden hochgradige Oedeme am ganzen Körper, starker Ascites und Hydrothorax. Die Atmung war durch letzteren ganz erheblich behindert. A ber auch nach der Entbindung wollten die Oedeme trotz salzfreier Diät, feuchter Packungen etc. nicht abnehmen. Erst als wir vom vierten Wochenbettstage ab die Wasserzufuhr auf ein Minimum beschränkten ( $800 \mathrm{ccm}$ pro die), trat eine längere Zeit anhaltende, enorme Harnflut ein, durch die dem Körper der Kranken innerhalb von $1 \frac{11}{2}$ Wochen etwa 16 Liter Wasser entzogen wurden (vgl. die folgende Tabelle).

$\begin{array}{ccc}\text { Wochenbettstag } & \begin{array}{c}\text { zugeführte } \\ \text { Flitissigkeitsmenge } \\ \text { ccm }\end{array} & \begin{array}{c}\text { Urinmenge } \\ \text { ccm }\end{array} \\ \text { l. } & ? & 270 \\ 2 . & 1150 & 1650 \\ 3 . & 925 & 1600 \\ 4 . & 770 & 3290 \\ 5 . & 800 & 2950 \\ 6 . & 800 & 3050 \\ 7 . & 800 & 2800 \\ 8 . & 800 & 2800 \\ 9 . & 800 & 2830 \\ 10 . & 800 & 2150 \\ 11 . & 800 & 1800\end{array}$

Eiweiß fand sich nach dieser Zeit nur noch in Spuren im Urin. Das Befinden der Patientin war jetzt dauernd ausgezeichnet.

Trotz der Wirksamkeit der eben skizzierten Behandlungsmethoden bleibt aber doch noch eine gewisse Anzahl von Fällen übrig, bei denen wir auf diese Weise nicht zum Ziele kommen und wo wir gezwungen sind, im Interesse des mütterlichen Lebens die künstliche Frühgeburt einzuleiten.

Ueber die Bedeutung dersalzarmen Diätbei der Be- 
handlung resp. Nachbehandlung der Eklampsie kann ich mich kurz fassen, denn es kommen hier im großen und ganzen dieselben Gesichtspunkte in Betracht, wie ich sie bereits bei der Besprechung der Nephritis gravidarum hervorhob. Erstes Erfordernis einer rationellen Eklampsiebehandlung bleibt eine möglichst rasche Entbindung; wir werden also im allgemeinen erst im Wochenbett Gelegenheit zur therapeutischen Verwendung der kochsalzarmen Diät finden. Hierbei haben wir wiederholt die Beobachtung gemacht, dab ein auffälliger Unterschied im Erfolg unserer Therapie bestand, je nachdem es sich um eine Erst- oder Mehrgebärende handelte. Bei den Erstgebärenden schwanden gewöhnlich die Krankheitserscheinungen der Eklampsie in kurzer Zeit, sodaß zum Beispiel von einer Albuminurie oft schon nach wenigen Tagen fast nichts mehr nachzuweisen war. Ganz anders bei Vielgebärenden. Hier trat nach einer erheblichen Besserung in den ersten Tagen post partum sehr bald ein gewisser Stillstand in der Rekonvaleszenz ein. Es blieb häufig eine Albuminurie von etwa $1-2 \%$ Albumen bestehen. Die Zylinder schwanden nicht völlig aus dem Urin, der Blutdruck war dauernd leicht erhöht, ohne daß dabei das Allgemeinbefinden der betreffenden Kranken erheblicher gestört gewesen wäre. Diese Differenz im klinischen Verlauf der einzelnen Eklampsien ist um so bemerkenswerter, als Ahlfeld in einer neuerdings erschienenen Arbeit die Eklampsie der Erstgebärenden von der der Vielgebärenden in bezug auf die Pathogenese der Krankheit scheidet. Während er die Ursache der letzteren in einer schon vor Beginn der Gravidität bestehenden chronischen Nierenschädigung vermutet (angeborene oder erworbene Defekte, alte Entzündungen, Schrumpfniere), hält er die Eklampsie der Erstgebärenden für die Folge einer Giftstauung, die veranlaft wird durch eine vorübergehende, mit der Schwangerschaft im engsten $\mathrm{Zu}$ sammenhang stehende Niereninsuffizienz (mechanische Schädigung, Leydensche Schwangerschaftsniere). Mit dieser Ahlfeldschen Einteilung der Eklampsien lassen sich unsere klinischen Beobachtungen einigermaßen in Einklang bringen, wobei es allerdings dahin gestellt bleiben mag, ob wir in einer primären Niereninsuffizienz tatsächlich die Causa morbi zu suchen haben.

\section{Cystitis. Pyelitis.}

Bei der Behandlung dieser Krankheiten spielt neben der medikamentösen Therapie die Diät eine grobe Rolle. Man verlangt von ihr, sie solle "reizlos" sein, und empfiehlt aus diesem Grunde die reine Milchdiät. Wie wir nun bereits oben hervorgehoben, läßt sich diese bei Erwachsenen nur schwer längere Zeit durchführen, und aus diesem Grunde bildet die salzarme Kost bei der Behandlung der Cystitis und Pyelitis einen wertvollen Ersatz für die reine Milchdiät.

Wir haben wiederholt bei den gerade in der Schwangerschaft sehr häufigen Pyelitiden und Cystitiden die NaCl-arme Diät verwendet und im allgemeinen recht zufriedenstellende Resultate erzielt. Ein auf diese Weise behandelter Pyelitisfall sei auch wegen des sonstigen klinischen Interesses, das er beansprucht, hier kurz erwähnt:

Eine an Nephritis gravidarum leidende Erstgebärende war durch Bettruhe, salzarme Diät etc. So gebessert worden, daß das normale Ende der Gravidität abgewartet werden konnte. Der Eiweißgehalt des Urins schwankte zwischen $3 \%$ und $4 \%$, die Zylindrurie war mäßig stark, das Allgemeinbefinden gut. Die Entbindung verlief normal. Das Kind wog bei der Geburt $3600 \mathrm{~g}$. Aus einer kurz ante partum durch Katheterisieren gewonnenen Urinprobe ließen sich zahlreiche Staphylokokkenkolonien auf der Agarplatte züchten. Therapie: Bettruhe, salzarme Diät, Urotropin, Blasentee.

Daraufhin besserten sich sämtliche Krankheitssymptome ganz erheblich. Die sieben Tage post partum vorgenommene Zystoskopie mit beiderseitigem Ureterenkatheterismus ergab folgendes Resultat: die Blasenschleimhaut zeigte leichte zystitische Veränderungen; beide Ureteren ließen sich unschwer katheterisieren. Der rechte Ureterurin war getrübt und enthielt zahlreiche Leukozyten, wenig Epithelien, einzelne Zylinder. Auf der Agarplatte gingen eine groBe Anzahl Staphylokokkenkolonien an. Der linke Ureterurin war klar. Im Sediment fanden sich Epithelien, wenig Leukozyten, einzelne Zylinder. Die Agarplatte blieb steril.

Es bestanden also nephritische Veränderungen an beiden Nieren; außerdem war eine rechtseitige Staphylokokkenpyelitis und eine leichte Cystitis vorhanden. Nach weiteren acht Tagen waren fast alle Krankheitssymptome verschwunden; nur ganz vereinzelte Zylinder ließen sich noch nachweisen. Der Urin erwies sich als steril. Es war uns also gelungen, nicht nur die bestehende Nephritis gravidarum bis auf eine minimale Zylindrurie zu beseitigen, sondern auch die durch Staphylokokkeninfektion bedingte Pyelitis und Cystitis völlig zu heilen.

Zusammenfassung. Die kochsalzarme Diät stellt durchaus kein Allheilmittel dar, mit dem jede Nephritis gravidarum mit Sicherheit gebessert oder gar geheilt werden könnte. Es gibt vielmehr Fälle schwerster Schwangerschaftsnephritis, wo diese Therapie ebenso wie jede andere konservative Behandlungsmethode versagt, wo wir gezwungen sind, zur Rettung des mütterlichen Lebens die künstliche Frühgeburt einzuleiten. Die Praxis hat jedoch gezeigt, daß die systematisch durchgeführte NaCl-Entziehung, ev. kombiniert mit Einschränkung der Flüssigkeitszufuhr, sehr häufig ein äußerst wirksames Mittel darstellt, nicht nur die Oedeme der Schwangerschaftsnephritis rasch zu beseitigen, sondern auch alle anderen Krankheitssymptome erheblich $\mathrm{zu}$ bessern. Bei der Behandlung der Schwangerschaftscystitis und -pyelitis kann die kochsalzarme Kost als „reizlose“ Diät neben der absoluten Milchdiät mit Erfolg Verwendung finden.

Literatur: Achard, Gazette des hôpitaux 1898. - Derselbe, Presse médicale 1901. - A hlfeld, Zeitschrift für Geburtshilfe und Gynäkologie 1908, Bd. 63. Birnbaum, Archiv für Gynäkologie 1907, Bd. 83. - Bittorf, Deutsches Archiv für klinische Medizin, Bd. 94. - Bittorf und Jochman n, Ebendaselbst, Bd. 89. Bohne, Fortschritte der Medizin 1897. - Cramer, Monatsschrift für Geburtshilfe Bohne, Fortschritte der Medizin 1897. - Cramer, Monatsschrift für Geburtshilfe
und Gynäkologie 1906, Bd. 23. - Derselbe, Münchener medizinische Wochenschrift und Gynäkologie 1906, Bd. 23. - Derselb e, Mänchener medizinische Wochenschrift
1907. - Strauss, Therapie der Gegenwart 1903. - Derselbe, Zeitschrift far physikalische und diätetische Therapie 1908. - Widal, Verhandlungen auf dem 26 . KongreB kalische und diätetische Therapie 1908. - W i dal, Verhandlungen auf dem 26. KongreB
für innere Medizin. Wiesbaden 1909. - Z a n geme is ter, Zeitschrift für Geburtshilfe und Gyaakologie 1903, Bd. 49 und 50. - Derselbe, Archiv für Gynäkologie 1908, und Gyose
Bd. 84 . 